\title{
Analysis of distributed generation system and the influence on voltage distribution
}

\author{
Tan Hongen, Wang Bo, Liu Dichen, Guo Suxuan \\ School of Electrical Engineering. Wuhan University, Wuhan, China \\ therp@yahoo.cn
}

Keywords: distributed generation system, micro-grid, voltage distribution

\begin{abstract}
As the requirement for the power quality is increasing nowadays, the distributed source energy has been widely used. The association of different Distributed Generation System (DGS) could cause as many problems as it may solve. Micro-grid associates the distributed generation and the loads as a subsystem. The generation and loads could separate from the distribution system to isolate the micro-grid's load from the disturbance without harming the distributed network's integrity. This paper discusses the associated operation of the distributed generation system and the influence it introduced into the distributed network. The design the distributed generation system would influence the voltage distribution of the system. Simulation and analysis has been done to verify it and the methods to avoid the harm it introduced to the network are proposed.
\end{abstract}

\section{Introduction}

The development of economy and technology has changed the demand and requirement for the electricity generation and transmission. The centralized generation facilities are giving way to smaller, more distributed generation partially due to the loss of traditional economies of scale. The distributed generation system has the following obvious advantages: 1) using the clean energy properly such as the solar energy, wind energy and biology fuel to reduce the emission of wasted gas during electricity generation; 2) in the distribution network, when the physical capacity of the generation system is insufficient as the increase of the loads, building the distributed generation system could satisfy the partial increase of loads and reduce the investment of electricity generation facilities; Meanwhile, it could make up the insufficiency of the peak of electricity consumption; 3 ) It could improve the partial reliability of power supply in the distributed network; 4) It could improve the efficiency of energy use and reduce the loss in transmission grid, such as using the technology of thermoelectricity cogeneration to enhance the utilization ratio of the primary energy.

However, the distributed generation system could cause the problems in the distributed network as much as it could solve. With the increase of permeability of distributed generation system, its development is limited by the harm it brought to traditional distributed system. The utilization of single machine distributed source has the problems of high cost and difficult control. On the other hand, distributed source is an uncontrolled source for the large power grid. Thus, the large power grid could use the method of limitation and isolation to control the distributed sources to reduce the impact for the large power grid. In 2001 the United States has proposed the standard IEEEP1547/D08--"Draft Standard for Interconnecting Distributed Sources with Electrical Power System", and designs the grid-connected standard for the distributed source: when there is any fault happens in the power grid, the distributed sources should shut down immediately, and it large limits the efficiency of the distributed sources. Also, the passive radicalized structure and the one-way single-path feature of the traditional distributed system has limited the operation of distributed generation system. They should be regarded as the load and the electricity they produced should be smaller than the loads, which limits the structure of the distributed generation system. The concept of micro-grid is proposed to solve the problems. Micro-grid is proposed in 2001 by R. H. Lasseter in University of Wisconsin-Madison. It is consisted by the distributed source such as photovoltaic cells and fuel cells, energy storage facilities, energy transformation facilities, monitoring and protecting facilities and relative loads. It is an isolating system which could realize the self-control, 
protection and management. The micro-grid could be operating connected with the distributed system, or isolated with the grid. In micro-grid, the electricity users need is provided by the wind power system, photovoltaic system, fuel cells and so forth. In the prerequisite of satisfying the thermal supply of the users, the micro-grid associates all kinds of distributed sources to provide electricity. Micro-grid could be controlled easily and is connected with the distributed grid. When there is fault happening in upstream network, micro-grid could operate isolated with the main grid. Viewing from the load side, micro-grid could provide the electricity for the thermal use and enhance the reliability of power supply, reduce the waste gas and improve the power quality by reducing the voltage sags and swells.

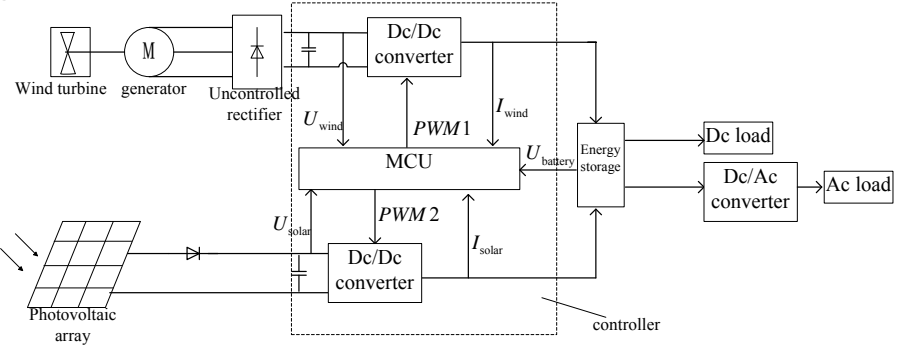

Fig. 1 the configure of the micro-grid

This paper introduces the associated operation of photovoltaic system and wind power system. The associated system is regards as a micro-grid in the power system. The voltage distribution is influenced by the distributed sources. Simulations are carried out to verify the results and methods to obtain a better voltage support are proposed.

\section{Influence of distributed generation system on power system}

The micro-grid includes the distributed sources, energy storage facilities and energy consumption loads. The associated distributed sources are designed to transform the unstable wind power and solar power to stable energy. In order to minimize the unbalance of power supply and consumption by the weather features, the storage battery is introduced to adjust and balance the energy. The loads include the ac consumption and dc consumption. Dc loads could be directly connected in the grid and the ac loads should be connected in the grid via the inverter. The structure of the micro-grid is shown in Fig.1.

An isolating associated distributed source system in this paper is consisted by the wind power system, photovoltaic system and energy storage facilities. The wind turbine in this system is directly coupled with the permanent magnet generator and provides three-phase ac power which has changing voltage and frequency. After the three-phase uncontrolled rectifier and LC output filter, the ac power could be changed into the dc power which has a changing voltage. The output dc voltage of wind power system is connected with the ac power supplying by the photovoltaic system and obtains the dc power satisfying the charging requirement of storage battery. The controller in the associated system has the functions as controlling the charging of wind power system and photovoltaic system to the storage battery, realizing the discharging of the storage battery to the load, sampling the parameters while the system operating and communication. The controller injects the dc power to the inverter and outputs the stable three-phase ac power to the distributed grid.

In the whole system, the associated distributed source system is connected into the distributed system as a micro-grid. As the associated system is seems as an entire and isolated system, the analysis and control for that is simpler. In this paper, the influence of the distributed generation system is discussed based on the micro-grid

The influence of the distributed generation system to distributed grid includes the design of distributed sources, the improvement of power quality and the monitoring and protection while the voltage sags and swells happens. The design of the distributed sources means the design of the location and capacity of the distributed sources in the micro-grid. The change of location and capacity could both change the voltage and current distribution in the distributed grid, thus 
influences the voltage and power loss of the whole distributed grid. Proper design of the distributed sources could bring positive influence to the distributed grid. The stable voltage could be maintained and the power loss could be reduced. The constant-power stable model is used in this paper. The distributed source is regarded as an equivalent current source and the load is considered as a node. The equivalent model of the distributed system is shown in Fig.2.

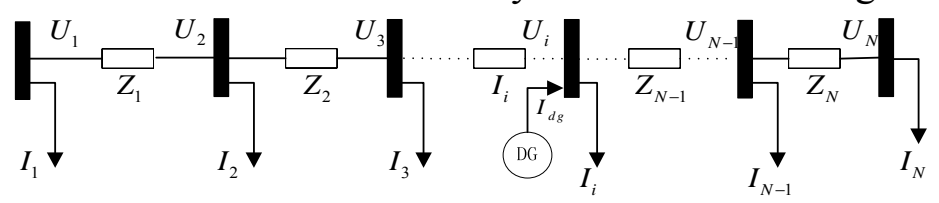

Fig.2 the equivalent model of the distributed system

The current distribution in the system is

$$
I_{d}(l)=\left\{\begin{array}{l}
\int_{l}^{L} I_{d}(x) d x-I_{D G}, 0 \leq l \leq M \\
\int_{l}^{L} I_{d}(x) d x, M \leq l \leq L
\end{array}\right.
$$

In this equation, $\mathrm{L}$ is the length of the feeder line; the distributed source is connected to the system M miles away from the bus of distributed grid. The impedance is $r(\Omega / \mathrm{m})$, and the function of the current distribution along the feeder line is presented by $I_{d}(l)$. The voltage distribution in the system is

$$
U(l)=E-\left\{\begin{array}{l}
\int_{0}^{l}\left(I_{d}(l)-I_{D G}\right) r d x, 0 \leq l \leq M \\
\int_{0}^{M}\left(I_{d}(l)-I_{D G}\right) r d x+\int_{M}^{L} I_{d}(l) r d x, M \leq l \leq L
\end{array}\right.
$$

The simplified model of the distributed system is shown in Fig.3.

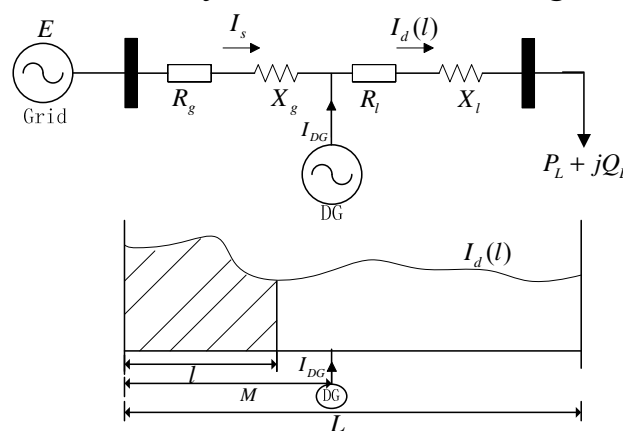

Fig. 3 the simplified model of the distributed system

As the voltage of the distributed grid, the impedance per mile and the current distribution is constant, thus the current which is injected by the distributed source and the location of them determine the voltage distribution in the grid.

\section{Simulation and analysis}

This paper uses PSASP to simulate the standard IEEE33 nodes model. This distributed grid is a medium voltage level grid. The basic voltage is $10 \mathrm{KV}$. As the load is relatively small, the basic capacity is chosen as 1MW. The standard IEEE33 nodes model is shown in Fig.4.

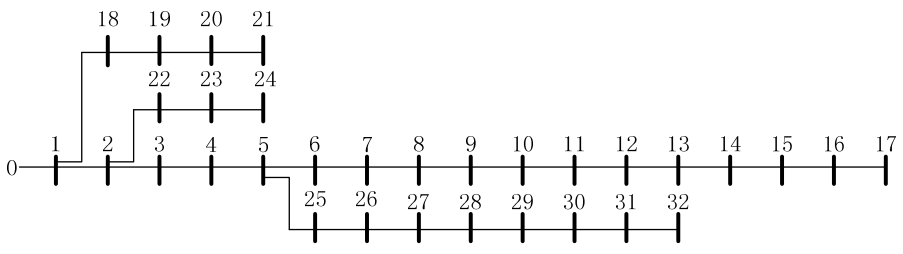

Fig.4 standard IEEE33 nodes model 
Simulate the voltage distribution of the grid without the distributed source, and choose five nodes exceeding the limitation series to connect in the distributed sources. The total capacity of the distributed sources is equal to the peak load power, which is $4.07 \mathrm{MW}$. Divide the power in average to the five selected nodes. Changing the location and the injected capacity of the distributed source and analyzes their influences to the voltage distribution in the grid. The voltage distribution of the system without the distributed source is shown in Fig.5.

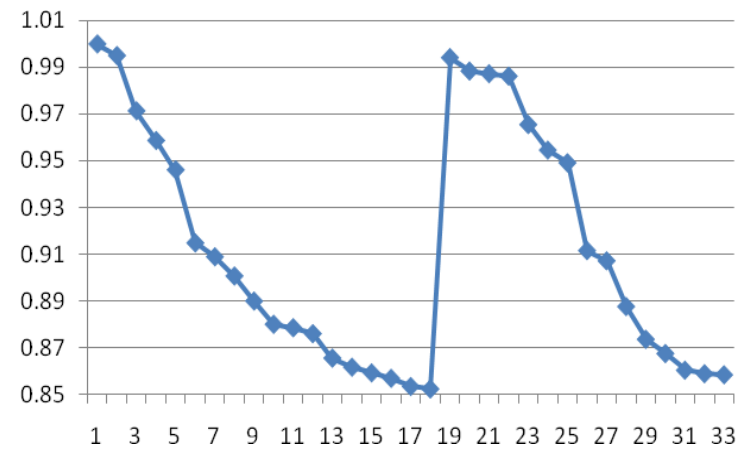

Fig. 5 the voltage distribution without the distributed source

When the centralized source is connected in the grid, the source in the terminal side of the distributed grid has a better effect than that of the other location. Thus, choose the following five topologies to do the simulation in PSASP, the simulation result is shown in Fig.6.

1) Node5 $11,17,28,32$

2) Node5 $11,16 、 28,32$

3) Node5,11,16、28,31

4) Node5 $11,15,28,30$

5) Node5、11、17、28、31

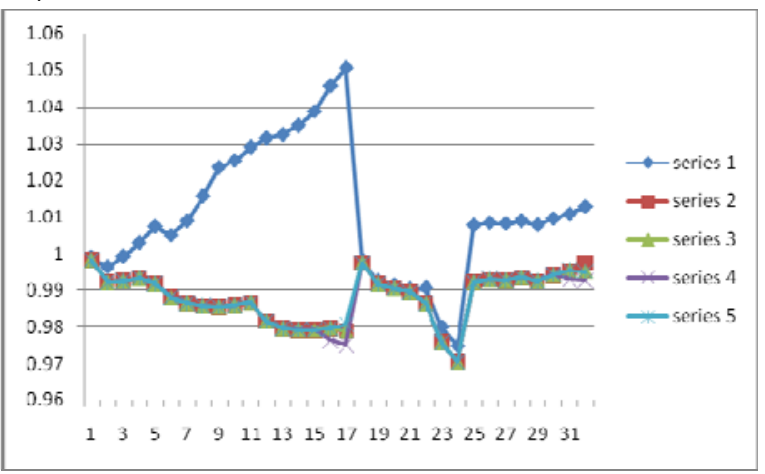

Fig. 6 the comparison of the five topologies of distributed sources

It could be seen from the Fig.6, except for the first condition that has a large voltage support, and the other four conditions are similar. Thus, connecting the distributed sources near the termination of the distributed grid could obtain a better effect for the voltage distribution.

As is discussed above, injecting the distributed sources at 5, 11, 16, 28, 31 nodes have an ideal effect for the voltage distribution. Changing the injecting capacity of each distributed source and keep the whole injecting capacity the same, the capacity distribution is as Table I and the simulation result is shown in Fig. 7.

TABLE I. capacity distribution of the distributed sources

\begin{tabular}{|c|c|c|c|c|c|}
\hline & Node 5 & Node 11 & Node 16 & Node 28 & Node 31 \\
\hline Series 1 & $100 \%$ & $30 \%$ & $70 \%$ & $100 \%$ & $100 \%$ \\
\hline Series 2 & $100 \%$ & $70 \%$ & $30 \%$ & $100 \%$ & $100 \%$ \\
\hline Series 3 & $100 \%$ & $100 \%$ & $100 \%$ & $30 \%$ & $70 \%$ \\
\hline Series 4 & $100 \%$ & $100 \%$ & $100 \%$ & $70 \%$ & $30 \%$ \\
\hline Series 5 & $100 \%$ & $70 \%$ & $100 \%$ & $30 \%$ & $100 \%$ \\
\hline
\end{tabular}




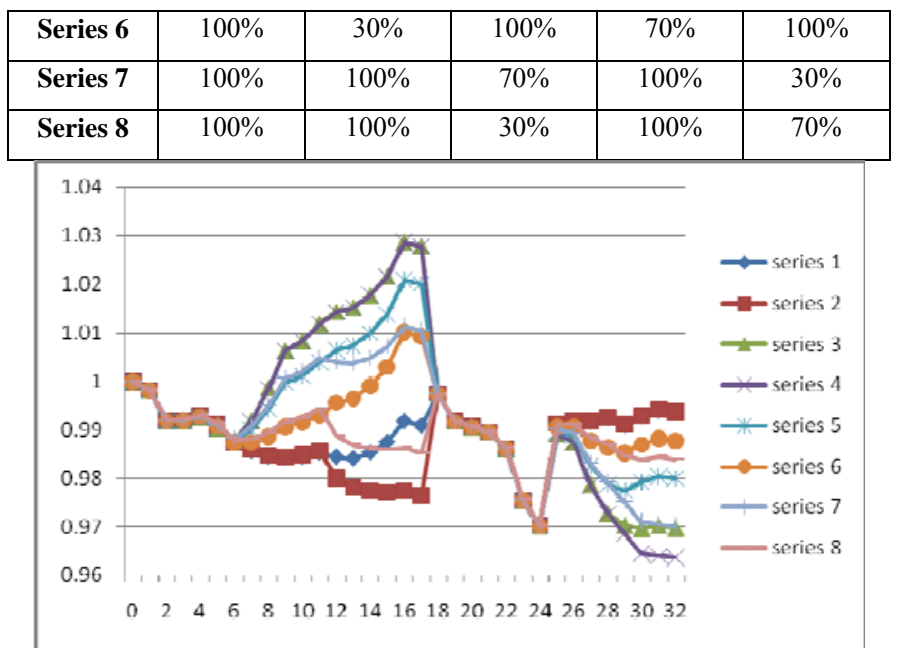

Fig.7 comparison of the different capacity distribution of the distributed sources

From Fig.7, connecting the distributed sources in the heavier line has a larger influence for the voltage distribution than that of the lighter line. When the total capacity of the distributed source remains the same, connecting the distributed sources near the termination of the grid could obtain better voltage support. Adjusting the capacity in the lighter line has small influence for the voltage distribution.

Thus the location and capacity of the distributed sources should be designed properly to obtain the stable voltage and better voltage support effect.

\section{Conclusion}

The distributed generation system would make the association of many kinds of distributed system possible and easy to control. However, the problems it connected into the grid would be as much as it solve, such as the voltage distribution. If the location and injecting capacity are not properly designed, the voltage could easily exceed the limitation and harm the stability of the system. This paper uses PSASP to simulate the various locations and injecting capacity of the distributed system and verify the proper location and capacity ratio of the distribution system. This method would improve the power quality and enhance the stability of the system.

\section{References}

[1] Yibo Wang, Jing Li, "Analysis of the maximum installed capacity of PV station constrained by power system security and stability", Solar Energy, Vol.29, No. 8, Aug. 2008

[2] Wang Zhiqun, Zhu shouzhen, "Study on Location and Penetration of Distributed Generations", Automation of Electric Power System, Vol. 17, No. 1, Feb. 2005

[3] Liang Caihao, Duan Xianzhong, "Distribution generation and its impaction on power system", Automation of Electric Power System, Jun. 2005

[4] M. N. Marwali, and A. Keyhani, "Control of Distributed Generation Systems", IEEE Transactions on power electronics, Vol. 19, No. 6, Nov. 2004 